\title{
Correlations between plasma noradrenaline concentrations, antioxidants, and neutrophil counts after submaximal resistance exercise in men
}

\author{
A Ramel, K-H Wagner, I Elmadfa
}

Br J Sports Med 2004;38:e22 (http://www.bjsportmed.com/cgi/content/full/38/5/e22)

Background: Generation of reactive oxygen species (ROS) during exercise has been linked to increased oxygen consumption. ROS could also be produced by other mechanisms - for example, a respiratory burst of neutrophils or catecholamine auto-oxidation-when oxygen consumption is only moderately increased.

Objectives: To investigate noradrenaline concentrations, neutrophil counts, plasma antioxidants, and lipid oxidation products before and after acute resistance exercise.

Methods: 17 male participants undertook a submaximal resistance exercise circuit (10 exercises; $75 \%$ of the one repetition maximum; mean (SD) exercise time, 18.6 (1.1) minutes). Blood samples were taken before and immediately after exercise and analysed for plasma antioxidants, noradrenaline, neutrophils, and lipid oxidation products. Wilcoxon's signed-rank test and Pearson's correlation coefficient were used for calculations.

Results: Neutrophils, noradrenaline, fat soluble antioxidants, and lipid oxidation products increased after exercise. Noradrenaline concentrations were associated with higher antioxidant concentrations. Neutrophils were related to higher concentrations of conjugated dienes.

Conclusions: Submaximal resistance exercise increases plasma antioxidants. This might reflect enhanced antioxidant defence in response to the oxidative stress of exercise, though this is not efficient for inhibiting lipid oxidation. The correlation between noradrenaline concentrations and plasma antioxidants suggests a modulating role of the stress hormone. Neutrophils are a possible source of oxidative stress after resistance exercise.
A cute exercise can induce oxidative stress ${ }^{12}$ and lower antioxidant status. ${ }^{3}$ When generation of reactive oxygen species (ROS) exceeds antioxidant capacity, lipids, proteins, and other cell components may be oxidised. ${ }^{4}$ Generation of ROS during exercise has been linked to increased oxygen consumption, ${ }^{4}$ but ROS could also be produced by alternative or additional mechanisms-for example, a respiratory burst of neutrophils or catecholamine auto-oxidation-when oxygen consumption is only moderately increased (such as in resistance exercise).

Our aim in this study was to determine, first, whether acute resistance exercise effects plasma antioxidants and lipid oxidation products, and second, whether changes in antioxidant concentrations after acute resistance exercise are related to neutrophil counts and noradrenaline concentrations.

\section{METHODS \\ Subjects}

A mixed sample of 17 male participants was included into the study (table 1). Ten participants were not resistance trained, while five reported performing endurance exercise-that is, running or bicycling two to three times a week. Seven participants were resistance trained, and five reported to practising endurance exercise two to three times a week as well.

The participants were informed about the purpose, nature, and potential risks and gave their written informed consent before participating. The study protocol was approved by the ethics committee of the Medical Faculty, University of Vienna.

\section{Experimental protocol}

One week before the start of the study, subjects were shown the 10 exercises of the resistance exercise circuit (bench press, leg press, latissimus dorsi pull, leg extension, shoulder press, triceps exercise, crunch, vertical row, biceps curl, and pull up) and their one repetition maximum (1-RM) for each exercise was evaluated. For the exercise crunch, the maximum number of repetitions was evaluated. The exercise intensity for the main test was defined individually at $75 \%$ of the 1 $\mathrm{RM}$; the mean number of repetitions was 9. For the exercise crunch, $75 \%$ of the maximum repetitions were done in the main test.

For the main test, subjects came to the resistance exercise circuit at 07.30 after an overnight fast and having abstained from alcohol since the day before. The participants had not done any exercise for two days before the experiment. After a warm up on a cycle ergometer (15 minutes, $75 \mathrm{~W}$ ), each subject carried out the submaximal resistance circuit exercise once at the defined intensity. The recovery time between the different exercise stations was set at one minute. The mean (SD) exercise time (without warm up) was 18.6 (1.1) minutes. A catheter was introduced into an antecubital vein shortly before the test. Blood samples were drawn 30 minutes before the resistance training circuit and immediately after.

\section{Biochemical analyses}

All measurements were made in duplicate. Fat soluble antioxidants ( $\alpha$ and $\gamma$ tocopherol, $\beta$ carotene, and lycopene) were measured by the method of Jacob and Elmadfa. ${ }^{5}$ Ascorbic acid was measured by the method of Denson and Bowers. ${ }^{6}$ Malondialdehyde (MDA) was measured according to Wong et al. ${ }^{7}$ Conjugated dienes of linoleic acid were measured by the method of Banni et al. ${ }^{8}$ Noradrenaline concentrations were measured using the method of Hollenbach et al. ${ }^{9}$ Neutrophils were counted with a blood

Abbreviations: MDA, malondialdehyde; ROS, reactive oxygen species; 1-RM, one repetition maximum 


\begin{tabular}{ll}
\hline $\begin{array}{l}\text { Table 1 } \\
(\mathrm{n}=17)\end{array}$ & Characteristics of the participants \\
\hline Variable & Mean (SD) \\
\hline Age (years) & $29.5(7.1)$ \\
Height (m) & $1.82(0.06)$ \\
Body mass (kg) & $82.1(7.6)$ \\
Body fat (\%) & $16.8(5.0)$ \\
BMI (kg/m $\left./ \mathrm{m}^{2}\right)$ & $24.8(1.8)$ \\
\hline BMl, body mass index. & \\
\hline
\end{tabular}

analyser in the department of haematology at the General Hospital Vienna. Plasma volume changes were calculated using the formula of Van Beaumont et al. ${ }^{10}$

\section{Statistical analyses}

Statistical analyses were done using SPSS/PC 10.0. The effect of exercise was tested using Wilcoxon's signed rank test. Correlations were calculated using Pearson's correlation coefficient $r$. Statistical significance was set at $\mathrm{p}<0.05$.

\section{RESULTS}

Neutrophils, noradrenaline concentrations, fat soluble antioxidants, MDA, and conjugated dienes all increased after exercise. Ascorbic acid decreased after exercise, although not significantly. Plasma volume decreased slightly after exercise $(-2.5(1.2) \%)$; correction for plasma volume therefore attenuated the changes in the investigated variables (table 2). There were no differences between resistance trained and untrained participants in the investigated variables before and after exercise.

The increase in noradrenaline concentrations after exercise correlated positively with the increase in $\gamma$ tocopherol $(r=0.584, \mathrm{p}=0.046), \beta$ carotene $(r=0.710, \mathrm{p}=0.007)$, and lycopene $(r=0.553, \mathrm{p}=0.050)$ after exercise. Ascorbic acid concentrations correlated positively with the increase in noradrenaline concentrations after exercise $(r=0.647$, $\mathrm{p}=0.031)$. The increase in neutrophils after exercise was related to conjugated dienes $(r=0.601, \mathrm{p}=0.018)$.

\section{DISCUSSION}

Acute resistance exercise increases lipid oxidation products, antioxidant concentrations, neutrophils, and noradrenaline concentrations in plasma, which cannot be explained by plasma volume changes. Despite mobilisation of fat soluble antioxidants during exercise, the plasma concentrations of MDA increased after exercise. This is in agreement with previous investigations involving resistance exercise, ${ }^{11}$ indicating inadequacies in the antioxidant defence system. The increase in $\gamma$ tocopherol, $\beta$ carotene, and lycopene concentrations during exercise correlated with the increase in noradrenaline. $\alpha$ Tocopherol, quantitatively the most important vitamin E vitamer, did not correlate with noradrenaline concentrations. It has been suggested that there is a rapid flux of $\alpha$ tocopherol between plasma and tissue, ${ }^{12}$ and this could explain why we could not detect any association between plasma noradrenaline and $\alpha$ tocopherol. There is a large variability in the response of plasma ascorbic acid to resistance circuit training and thus no significant differences between before and after the exercise, but higher ascorbic acid concentrations were also associated with the increase in noradrenaline concentrations.

The association between noradrenaline concentrations and antioxidants could represent a physiological mechanism ensuring adequate antioxidant defence during physical activity. This type of study and statistical analysis do not prove causality between noradrenaline and antioxidant concentrations; the observed correlations could also reflect the possibility that both factors-noradrenaline and antioxidants-respond independently of each other to exercise. However, it has been speculated that the release of catecholamines leads to upregulation of antioxidant defence. ${ }^{13}$ Catecholamine secretion parallels secretion of ascorbic acid from the adrenal gland, ${ }^{14}$ and there is research suggesting that noradrenaline secretion induces antioxidant defencefor example, superoxide dismutase expression. ${ }^{13}$

In our study no significant correlation between the stress hormone and the lipid oxidation products conjugated dienes and MDA could be observed after exercise, although it has been suggested that auto-oxidation of catecholamines, which generates a superoxide anion radical, could result in lipid oxidation. ${ }^{15}$ During short term resistance exercise, noradrenaline concentrations increase only moderately. We cannot rule out the possibility that during intensive endurance exercise, high noradrenaline concentration may contribute to oxidative stress. After short time resistance exercise there are other possible sources of free radicals causing lipid oxidation. ${ }^{16}$ For example, in our study there was a positive correlation between the neutrophil count and the concentrations of conjugated dienes; it is well known that neutrophils can

\section{Take home message}

- Acute submaximal resistance exercise increases absolute plasma antioxidant concentrations. This is not efficient at inhibiting the increase in lipid oxidation products in plasma.

- Noradrenaline concentrations are associated with higher plasma antioxidant concentrations, which could reflect a modulating role of the stress hormone.

Table 2 Plasma antioxidants, noradrenaline, neutrophils, and lipid oxidation products before and after exercise $(n=17)$

\begin{tabular}{|c|c|c|c|c|c|}
\hline & & Before exercise & After exercise & p Value & p Value* \\
\hline Noradrenaline & (nmol/l) & $1.46(0.53)$ & $2.32(0.90)$ & 0.006 & 0.006 \\
\hline Neutrophils & (cells $\times 10^{3} \mathrm{ml}$ ) & $2.66(1.01)$ & $3.83(1.48)$ & 0.001 & \\
\hline Ascorbic acid & ( $\mu \mathrm{mol} / /$ plasma) & $50.6(32.1)$ & $43.6(25.2)$ & 0.363 & 0.099 \\
\hline$\alpha$ Tocopherol & ( $\mu \mathrm{mol} / \mathrm{I}$ plasma) & $17.2(4.8)$ & $19.6(6.1)$ & 0.026 & 0.156 \\
\hline$\gamma$ Tocopherol & ( $\mu \mathrm{mol} / \mathrm{I}$ plasma) & $1.17(0.44)$ & $1.32(0.50)$ & 0.017 & 0.020 \\
\hline$\beta$ Carotene & ( $\mu \mathrm{mol} / /$ plasma) & $0.235(0.138)$ & $0.280(0.154)$ & 0.004 & 0.011 \\
\hline Lycopene & ( $\mu \mathrm{mol} / /$ plasma) & $0.210(0.159)$ & $0.241(0.153)$ & 0.023 & 0.061 \\
\hline MDA & ( $\mu \mathrm{mol} / /$ plasma) & $1.90(0.98)$ & $2.28(0.69)$ & 0.034 & 0.049 \\
\hline Conjugated dienes & (mg/l plasma) & $10.7(5.2)$ & $12.6(7.1)$ & 0.036 & 0.069 \\
\hline
\end{tabular}

Values are mean (SD)

*After correction for plasma volume changes. 
generate ROS through an oxidative burst, which might worsen exercise induced damage ${ }^{17}$ and contribute to lipid peroxidation. $^{18}$

\section{Authors' affiliations}

A Ramel, Unit for Nutrition Research, University of Iceland, Reykjavik, Iceland

K-H Wagner, I Elmadfa, Institute of Nutritional Sciences, University of Vienna, Vienna, Austria

Correspondence to: Dr Alfons Ramel, Haedargardur 18, Reykjavik, Iceland; ramel@hi.is

Accepted 29 August 2003

\section{REFERENCES}

1 Sanchez-Quesada JL, Holms-Serradesanferm R, Serrat-Serrat J, et al Increase of LDL susceptibility to oxidation occurring after intense, long duration aerobic exercise. Atherosclerosis 1995; 118:297-305.

2 Witt EH, Reznick AZ, Viguie CA, et al. Exercise, oxidative damage and effects of antioxidant manipulation. J Nutr 1992;122:766-73.

3 Subudhi AW, Davis SL, Kipp RW, et al. Antioxidant status and oxidative stress in elite alpine ski racers. Int J Sport Nutr Exerc Metab 2001;11:32-41.

4 Ji LL. Free radicals and antioxidants in exercise and sports. In: Garrett WE, Kirkendall DT, eds. Exercise and sport science. Philadelphia: Lippincott Williams and Wilkins, 2000:299-317.

5 Jakob E, Elmadfa I. Rapid HPLC-assay of vitamin K1, A, E and beta-carotene status in children (7-19 years). Int I Vit Nutr Res 1995;65:31-5.
6 Denson KW Bowers EF. The determination of ascorbic acid in white blood cells. Clin Sci (Colch) 1961;21:157-62.

7 Wong SHY, Knight JA, Hopfer SM, et al. Lipoperoxides in plasma measured by liquid chromatographic separation of malondialdehyde-thiobarbituric acid adduct. Clin Chem 1987;33:214-20.

8 Banni S, Lucchi L, Baraldi A, et al. No direct evidence of increased lipid peroxidation in hemodialysis patients. Nephron 1996;72:177-83.

9 Hollenbach E, Schulz C, Lehnert H. Rapid and sensitive determination of catecholamines and the metabolite 3-methoxy-4-hydroxyphen-ethyleneglycol using HPLC following novel extraction procedures. Life Sci using HPLC following

10 Van Beaumont W, Greenleaf JE, Juhos L. Disproportional changes in hematocrit, plasma volume, and proteins during exercise and bed rest. J Appl Physiol 1972;33:55-61.

11 McBride JM, Kraemer WJ, Triplett-McBride T, et al. Effect of resistance exercise on free radical production. Med Sci Sports Exerc 1998;30:67-72.

12 Hutler M, Woweries S, Leithauser R, et al. Exercise-induced changes in blood levels of alpha-tocopherol. Eur J Appl Physiol 2001;85:151-6.

13 Mehta J, Li D. Epinephrine upregulates superoxide dismutase in human coronary artery endothelial cells. Free Radic Biol Med 2001;30:148-53.

14 Ji LL. Oxidative stress during exercise: implication of antioxidant nutrients. Free Radic Biol Med 1995; 18:1079-86.

15 Rodriguez-Martinez MA, Garcia-Cohen EC, Briones A, et al. Changes in plasma oxidative state with age and their influence on contractions elicited by noradrenaline in the rat tail artery. Life Sci 1999;65:915-24.

16 Smith LL, Miles MP. Exercise-induced muscle injury and inflammation. In: Garrett WE, Kirkendall DT, eds. Exercise and sport science, chapter 27.Philadelphia, Lippincott Williams and Wilkins, 2000:401-11.

17 Dallegri F, Ottonello L. Tissue injury in neutrophilic inflammation. Inflamm Res 1997:46:382-91.

18 Heinecke JW. Tyrosyl radical production by myeloperoxidase: a phagocyte pathway for lipid peroxidation and dityrosine cross-linking of proteins. Toxicology 2002;177:11-22. 